EPJ manuscript No.

(will be inserted by the editor)

\title{
Photoconductance of a one-dimensional quantum dot
}

\author{
M. Vicari, A. Braggio, E. Galleani d'Agliano, M. Sassetti \\ Dipartimento di Fisica, INFM, Via Dodecaneso 33, I-16146 Genova, Italy \\ Received: 22 October 2001 / Revised version: date
}

\begin{abstract}
The ac-transport properties of a one-dimensional quantum dot with non-Fermi liquid correlations are investigated. It is found that the linear photoconductance is drastically influenced by the interaction. While for weak interaction it shows peak-like resonances, in the strong interaction regime it assumes a step-like behavior. In both cases the photo-transport provides precise informations about the quantized plasmon modes in the dot. Temperature and voltage dependences of the sideband peaks are treated in detail. Characteristic Luttinger liquid power laws are found.
\end{abstract}

PACS. 71.10.Pm Fermions in reduced dimensions (anyons,composite fermions, Luttinger liquid, etc.) 73.63.kv Quantum dots - 73.50.Pz Photoconduction and photovoltaic effects

\section{Introduction}

In the last years, theoretical and experimental efforts have focused on the analysis and control of transport in low dimensional quantum systems. The experimental realization of one-dimensional (1D) quantum wires has opened new possibilities to investigate the influences of interactions and impurities on electron transport in the quantum regime [1,2.3]. Decreasing the electron density in the cleaved-edge-overgrowth GaAs/AlGaAs quantum wire, it was possible to reach the region of Coulomb blockade where transport occurs by transferring exactly one electron through a $1 D$ quantum island created by random impurities 4. The temperature dependence of the conductance Coulomb peak exhibited, for the first time, a clear ' evidence of a non-Fermi liquid behavior. Similar behaviors were also indicated by recent results of Raman scattering, which strongly suggests that semiconductor quantum wires are very probably dominated by non-Fermi liquid excitations $[5]$.

The existing broad theoretical understanding of 1D interacting electrons [6, 7,8] provided in the past quantitative results for dc-transport in the presence of correlations and impurities. 1D quantum dots embedded in a Luttinger liquid were subject of several theoretical investigations 9, 10, 11, 12, 13, 14. Microscopic charging effects and transport properties in the sequential and cotunneling regimes were discussed. Important results, connected with finite range interactions and spin dynamics were also obtained [15, 16.

Recently, frequency and time dependent effects have been investigated intensively. When an ac-field is applied, the conduction properties of the system can be strongly modified. Indeed an ac-polarization enables to conduct through new photon-assisted channels due to inelastic scattering of electrons and photons. The effect of the above photon assisted tunneling (PAT) becomes crucial in the presence of a quantum dot. Here, the electrons overcome the Coulomb blockade regime by absorbing photons from the external field. These additional transport channels enrich the conductance structure and give precise information about ground state and excited states in the dot.

PAT is a topic studied since about forty years. A paradigmatic result was obtained by Tien and Gordon [17 for a superconducting tunnel junction. Resonant tunneling in a periodic time-dependent external field through a double barrier was investigated within the framework of classical perturbation theory in order to examine the effects due to the interplay between the lifetime of an electron trapped in the well and the microwave period $[18]$. PAT has been investigated for $2 \mathrm{D}$ quantum dots in the region of charging effects and intra-dot transitions both in theoretical 19, 20 and experimental works [21, 22, 23, 24, 25. In the mean-field approximation, self-consistent renormalization of the driving field has been also studied 26. More recently PAT has been analyzed to determine the complex microwave photoconductance of a single quantum dot [27], but a great interest has also focused on systems formed by weakly coupled quantum dots [28]. In spite of this broad researches, mainly devoted to $2 \mathrm{D}$ systems, there is still a lack of investigations related to $1 \mathrm{D}$ quantum dots in the presence of ac-fields. Here, a microspic treatment of the interplay between correlation and ac-fields is crucial and can yield new information on the physics of non-Fermi liquids. In the past, ac-transport in a Luttinger liquid through a single barrier has been considered, including higher harmonics generation, frequency scaling properties and local fields corrections [29 30.31].

In this paper we present results of time dependent transport through a double barrier. We evaluate the pho- 
to-conductance of a $1 \mathrm{D}$ quantum dot as a response to a monochromatic signal in the sequential tunneling regime. The electron interactions are treated within the framework of the Luttinger liquid. We show that the ac-conduction features are strongly affected by the strength of the interaction. The latter gives rise to two particular regimes of strong and weak interaction. The difference between the two appears in the temperature and/or gate voltage dependence of the photoconductance. By varying the frequency we identify sideband peaks related to the ground and the excited states. The latter are due to the collective quantized plasmons in the dot. Non-analytic power laws of the peaks are also predicted and their dependence on the interaction is considered.

The paper is organized as follows. In Section 2 we describe the model. The $1 \mathrm{D}$ quantum dot is confined inside the single channel quantum wire by two high localized scattering barriers. Transport is tuned by gate and sourcedrain voltages. In Section 3 we present the solution for actransport in the regime of high frequencies. The current is described by a master equation [32,33, 14, generalized to the case of time-dependent polarizations. Section 4 is devoted to the discussion of the behavior of the linear photoconductance. In Section 5 we draw conclusions and discuss also the the consistency of the approximations.

\section{The model}

We consider 1D spinless interacting electrons treated via the bosonization technique [8]. The quantum dot is described by two symmetric delta-like barriers $V_{\mathrm{B}} \delta\left(x-x_{i}\right)$ at $x_{i}(i=1,2)$ where $x_{1}<x_{2}$. External dc- and ac-potentials are coupled to the dot in order to induce transport. The Hamiltonian is

$$
H=H_{0}+H_{\mathrm{B}}+H_{\mathrm{V}}
$$

The first term describes the collective low-energy charge density modes of the 1D electron gas $(\hbar=1)$, 6, 8

$$
H_{0}=\frac{v_{\mathrm{F}}}{2} \int_{-\infty}^{+\infty} d x\left[\Pi^{2}(x)+\frac{1}{g^{2}}\left(\partial_{x} \Theta(x)\right)^{2}\right] .
$$

Their quantization is realized by the field operator $\Theta(x)$ and its conjugate $\Pi(x)$. The former represents the longwavelength part of the electron number density

$$
\rho(x)=\rho_{0}+\sqrt{\frac{1}{\pi}} \partial_{x} \Theta(x),
$$

with the mean electron density $\rho_{0}=k_{\mathrm{F}} / \pi\left(k_{\mathrm{F}}\right.$ Fermi wave number). The velocity of the charge modes is renormalized with respect to the Fermi velocity $v_{\mathrm{F}}$, because of the electron interaction. It is $v=v_{\mathrm{F}} / g$, with the interaction strength

$$
\frac{1}{g}=\sqrt{1+\frac{V(q \rightarrow 0)}{\pi v_{\mathrm{F}}}}
$$

Here, $V(q)$ is the Fourier transform of the 3D Coulomb interaction projected along the wire [5].

The contribution of the two localized impurities involves $2 k_{\mathrm{F}}$-backscattering interference between left and right moving electrons. It can be written in the bosonized form as 10,11

$$
H_{\mathrm{B}}=U_{\mathrm{B}} \cos \left(\pi N_{+}\right) \cos \left[\pi\left(n_{0}+N_{-}\right)\right],
$$

where $U_{\mathrm{B}} \equiv \rho_{0} V_{\mathrm{B}}$, and $N_{ \pm}=\left[\Theta\left(x_{2}\right) \pm \Theta\left(x_{1}\right)\right] / \sqrt{\pi}$. The quantity $N_{+} / 2$, is associated with the unbalanced particles between left and right leads, while $N_{-}$represents the fluctuations of the particle number in the dot with respect to the mean electron number $n_{0}=\rho_{0} d ;\left(d=x_{2}-x_{1}\right)$.

The external fields consist of a dc-source-drain voltage $V$ that drops symmetrically at the barrier, and a gate voltage, renormalized by the ratio between gate and total capacitance, with a monochromatic component $V_{\mathrm{g}}+$ $V_{\mathrm{ac}} \cos (\omega t)$. The coupling is then $(e>0)$

$$
H_{\mathrm{V}}=-e\left[\frac{V}{2} N_{+}+\left(V_{\mathrm{g}}+V_{\mathrm{ac}} \cos (\omega t)\right) N_{-}\right]
$$

In the presence of ac-fields the particle current depends on the spatial position along the wire [34]. In the stationary limit $(t \rightarrow \infty)$ it will be given by a superposition of all harmonics of the monochromatic signal

$$
I_{\mathrm{st}}(x, t)=\sum_{l=-\infty}^{+\infty} I^{(l)}(x) e^{-i l \omega t} .
$$

Moreover, there will be displacement contributions that eventually will renormalize the total current [26].

In the following we will focus only on the steady component $(l=0)$ of the total current. This term is dominated by the particle current and allows us to neglect displacement contributions. It is space independent and can then be evaluated from the time derivative of the number of transferred particles through the dot

$$
I(t)=\frac{e}{2}<\dot{N}_{+}(t)>.
$$

The bracket $\langle\ldots\rangle$ includes a thermal average over the collective charge density modes away from the barriers and a statistical average over the final states of the reduced density matrix for the degrees of freedom at $x=x_{1}, x_{2}$. The former play the role of a thermal bath. They act as a dissipative source for the degrees of freedom at the positions of the barriers and they can be exactly traced out. The explicit contribution of their integration consists of two terms 34.

The first term is frequency independent and it is responsible to suppress changes in the particle number on the island, with respect to the equilibrium value. The corresponding energy is $E_{\mathrm{c}} N_{-}^{2}$ with the charge addition energy

$$
E_{\mathrm{c}}=\frac{\pi v_{\mathrm{F}}}{2 d g^{2}}
$$


Without interaction $(g=1)$, the addition energy is still finite $E_{\mathrm{c}}=\pi v_{\mathrm{F}} / 2 d=E_{\mathrm{P}}$, due to the Pauli principle, and the discreteness of the dot levels. On the other hand, for strong Coulomb interaction $E_{\mathrm{c}} \propto V(q \rightarrow 0) \gg E_{\mathrm{P}}$.

The second term is frequency dependent and represents the dynamical effects of the external leads and of the correlated excited states in the dot. Its influence is described by the spectral density [10,11]

$$
J(\omega)=\frac{\omega}{g}\left[1+\varepsilon \sum_{m=1}^{\infty} \delta(\omega-m \varepsilon)\right] \equiv J_{\text {leads }}(\omega)+J_{\mathrm{dot}}(\omega) .
$$

The first term is due to the leads, while the second reflects the discretization energy $\varepsilon$ of the charge modes in the quantum dot

$$
\varepsilon=\frac{\pi v_{\mathrm{F}}}{d g} \equiv 2 g E_{\mathrm{c}}
$$

\section{AC-transport}

For obtaining the time dependent current we have to consider the dynamics of the $N_{ \pm}$-variables under the influences of the external fields (河), the dissipation (10) and the $2 \mathrm{D}$ periodic potential (5). For high barriers $U_{\mathrm{B}} \gg E_{\mathrm{c}}$, the dynamics is dominated by tunneling events between nearest-neighbored minima, with amplitude $\Delta$ that is related to $U_{\mathrm{B}}$ via WKB-approximation. In the following, we will consider the contribution of uncorrelated hops off and onto the island (sequential tunneling processes) with the temperature smaller then the discretization energy $\varepsilon$.

The main quantities, in this case, are the tunneling rates. We identify with $\Gamma\left(U_{\nu, s}(n), t\right)$ the rate of hops through the right $(\nu=\mathrm{r})$ or left $(\nu=1)$ barrier, in the forward $(s=\mathrm{f})$ or backward $(s=\mathrm{b})$ direction, respectively with initially $n \equiv n_{0}+N_{-}$number of electrons in the dot. To the lowest order in the tunneling matrix element $\Delta$, one has 30

$$
\Gamma\left(U_{\nu, s}(n), t\right)=\int_{0}^{t} d t^{\prime} \kappa_{\nu, s}\left(n, t, t^{\prime}\right)
$$

where

$$
\begin{aligned}
\kappa_{\nu, s}\left(n, t, t^{\prime}\right)= & \frac{\Delta^{2}}{4} \exp \left[-W\left(t-t^{\prime}\right)+i U_{\nu, s}(n)\left(t-t^{\prime}\right)\right. \\
& \left.-i e \chi_{\nu, s} V_{\mathrm{ac}} \int_{t^{\prime}}^{t} d \tau \cos (\omega \tau)\right]+ \text { c.c. }
\end{aligned}
$$

Here $\chi_{\nu, \mathrm{f}}=-\chi_{\nu, \mathrm{b}}= \pm 1$ for $\nu=\mathrm{r}, 1$. The function $W(t)$ describes the influence of bulk modes, it is directly connected to the spectral density (10) by [30] $\left(\beta=1 / k_{\mathrm{B}} T\right)$,

$$
W(t)=\int_{0}^{\infty} d \omega \frac{J(\omega)}{\omega^{2}}\left[(1-\cos \omega t) \operatorname{coth} \frac{\beta \omega}{2}+i \sin \omega t\right] .
$$

The energies $U_{\nu, s}(n)$ are the differences between initial and final addition and electrostatic energies associated with different types of hops $(\nu, s)$

$$
\begin{aligned}
U_{\nu, s}(n) & =2 E_{\mathrm{c}}\left[\chi_{\nu, s}\left(n-n_{\mathrm{g}}\right)-\frac{1}{2}\right]-\eta_{s} \frac{e V}{2}, \\
n_{\mathrm{g}} & \equiv n_{0}+\frac{e V_{\mathrm{g}}}{2 E_{\mathrm{c}}} .
\end{aligned}
$$

The reference particle number $n_{\mathrm{g}}$ is defined by the gate voltage, and $\eta_{s}= \pm 1$ for $s=\mathrm{f}, \mathrm{b}$. The lack of timeinvariance due to the ac-field is reflected in the doubletime dependence of the kernel $\kappa\left(t, t^{\prime}\right)$. In the absence of the ac-field, $\kappa\left(t, t^{\prime}\right)$ is translational invariant and the corresponding dc-rate is the $t \rightarrow \infty$ limit of (12) for $V_{\mathrm{ac}}=0$

$$
\Gamma_{\mathrm{dc}}\left(U_{\nu, s}(n)\right)=\frac{\Delta^{2}}{4} \int_{-\infty}^{+\infty} d t e^{-W(t)} e^{i U_{\nu, s}(n) t}
$$

This integral can be explicitely evaluated at low temperatures, $k_{\mathrm{B}} T \ll \varepsilon$, 14

$$
\Gamma_{\mathrm{dc}}(U)=\sum_{p=-\infty}^{\infty} w_{p}(\varepsilon) \gamma^{(2 g)}(U-p \varepsilon)
$$

where $\gamma^{(g)}(U)$ is the tunneling rate of a single barrier

$$
\begin{aligned}
\gamma^{(g)}(U)= & \frac{\Delta^{2}}{4 \omega_{\mathrm{c}}} \frac{e^{\beta U / 2} e^{-|U| / \omega_{c}}}{\boldsymbol{\Gamma}(2 / g)}\left(\frac{\beta \omega_{\mathrm{c}}}{2 \pi}\right)^{1-2 / g} \\
& \times\left|\boldsymbol{\Gamma}\left(\frac{1}{g}+\mathrm{i} \frac{\beta U}{2 \pi}\right)\right|^{2}
\end{aligned}
$$

with frequency cutoff $\omega_{\mathrm{c}}$ and $\boldsymbol{\Gamma}(z)$ the Gamma function. The weights $w_{p}(\varepsilon)$, at discrete energies $\varepsilon$, are for $k_{\mathrm{B}} T \ll \varepsilon$

$$
w_{p}(\varepsilon)=\theta(p)\left(1-e^{-\varepsilon / \omega_{c}}\right)^{1 / g} \frac{\boldsymbol{\Gamma}(1 / g+p)}{p ! \boldsymbol{\Gamma}(1 / g)} e^{-p \varepsilon / \omega_{c}} .
$$

In the presence of the ac-field, the kernel $\kappa\left(\tau, \tau-\tau^{\prime}\right)$ depends periodically on $\tau$ with a period $\mathcal{T}=2 \pi / \omega$. This allows to write the stationary $(t \rightarrow \infty)$ rate $(12)$ in terms of principal and higher harmonics of the monochromatic field

$$
\Gamma_{\mathrm{st}}\left(U_{\nu, s}(n), t\right)=\sum_{l=-\infty}^{+\infty} \Gamma^{(l)}\left(U_{\nu, s}(n)\right) e^{-i l \omega t} .
$$

In the sequential tunneling regime the expression for the corresponding current is characterized by the sum over all possible contributions stemming from different electron numbers $n$ in the island, weighted by the occupation probability $p(n, t)$

$$
I(t)=\frac{e}{2} \sum_{n=-\infty}^{+\infty} \int_{0}^{t} d t^{\prime} p\left(n, t^{\prime}\right)\left[\kappa_{\mathrm{b}}\left(n, t, t^{\prime}\right)-\kappa_{\mathrm{f}}\left(n, t, t^{\prime}\right)\right],
$$


where $\kappa_{s}=\kappa_{\mathrm{r}, s}+\kappa_{1, s}$. The probability $p(n, t)$ is determined by the non-Markovian master equation [35]

$$
\begin{aligned}
& \dot{p}(n, t)=-\int_{0}^{t} d t^{\prime}\left\{p\left(n, t^{\prime}\right)\left[\kappa_{\mathrm{f}}\left(n, t, t^{\prime}\right)+\kappa_{\mathrm{b}}\left(n, t, t^{\prime}\right)\right]\right. \\
& -p\left(n+1, t^{\prime}\right)\left[\kappa_{\mathrm{l}, \mathrm{b}}\left(n+1, t, t^{\prime}\right)+\kappa_{\mathrm{r}, \mathrm{f}}\left(n+1, t, t^{\prime}\right)\right] \\
& \left.-p\left(n-1, t^{\prime}\right)\left[\kappa_{\mathrm{r}, \mathrm{b}}\left(n-1, t, t^{\prime}\right)+\kappa_{\mathrm{l}, \mathrm{f}}\left(n-1, t, t^{\prime}\right)\right]\right\} .
\end{aligned}
$$

Because of the ac-fields, the asymptotic solution of (22) will consist of a superposition of all harmonics

$$
p_{\mathrm{st}}(n, t)=\sum_{l=-\infty}^{+\infty} p^{(l)}(n) e^{-i l \omega t} .
$$

Inserting this expansion in the above master equation one obtains an infinite set of linear equations in which the $p^{(l)}(n)$ are coupled with each other via the $\kappa_{\nu, s}$ kernels. These equations can be solved recursively. For details see the similar discussion performed for a driven dissipative many states system [35].

Here we are interested in the limit of high frequencies. For $\omega \gg\left\{\Gamma^{(0)}\left(U_{\nu, s}(n)\right), \Delta, e V\right\}$, the ac-field oscillates too fast to be able to catch the details of the dynamics within one period $\mathcal{T}$. This corresponds to the non-adiabatic regime where each electron experiences many cycles of the ac-field during its presence inside the dot. In this limit one can approximate the kernels $\kappa_{\nu, \mathrm{s}}\left(m, t, t^{\prime}\right)$ in (22) by a time average [35]

$$
\begin{aligned}
& \kappa_{\nu, \mathrm{s}}\left(m, t, t^{\prime}\right) \approx \kappa_{\nu, \mathrm{s}}^{(0)}\left(m, t-t^{\prime}\right)= \\
& =\frac{1}{\mathcal{T}} \int_{0}^{\mathcal{T}} d \tau \kappa_{\nu, \mathrm{s}}\left(m, \tau, \tau-\left(t-t^{\prime}\right)\right),
\end{aligned}
$$

reducing the master equation (22) to a convolutive form. This implies a stationary solution without periodic oscillations as in (23). It can be written in terms of the lowest Fourier component $p^{(0)}(n)$ that satisfies detailed balance

$$
\begin{aligned}
& p^{(0)}(n)\left[\Gamma^{(0)}\left(U_{\mathrm{l}, \mathrm{f}}(n)\right)+\Gamma^{(0)}\left(U_{\mathrm{r}, \mathrm{b}}(n)\right)\right]= \\
= & p^{(0)}(n+1)\left[\Gamma^{(0)}\left(U_{\mathrm{l}, \mathrm{b}}(n+1)\right)+\Gamma^{(0)}\left(U_{\mathrm{r}, \mathrm{f}}(n+1)\right)\right],
\end{aligned}
$$

with the normalization constraint $\sum_{n=-\infty}^{+\infty} p^{(0)}(n)=1$. The average rate

$$
\Gamma^{(0)}\left(U_{\nu, \mathrm{s}}(m)\right)=\int_{0}^{\infty} d \tau \kappa_{\nu, \mathrm{s}}^{(0)}(m, \tau),
$$

represents the Fourier component corresponding to $l=0$ of the asymptotic rate (20). From the periodicity of the external field it can be written as a superposition of the dc-rate $\Gamma_{\mathrm{dc}}$ evaluated at integer multiples of the frequency $\omega 31$.

$$
\Gamma^{(0)}\left(U_{\nu, s}(n)\right)=\sum_{k=-\infty}^{+\infty} J_{k}^{2}\left(\frac{e V_{\mathrm{ac}}}{\omega}\right) \Gamma_{\mathrm{dc}}\left(U_{\nu, s}(n)+k \omega\right),
$$

here $J_{k}(x)$ is the Bessel function of order $k$.

Performing equivalent high frequency approximations on the kernels (21), one obtains the asymptotic and steady component $(l=0)$ of the current (7)

$$
\begin{aligned}
I^{(0)}= & \frac{e}{2} \sum_{n=-\infty}^{+\infty} p^{(0)}(n)\left[\Gamma^{(0)}\left(U_{\mathrm{l}, \mathrm{b}}(n)\right)+\Gamma^{(0)}\left(U_{\mathrm{r}, \mathrm{b}}(n)\right)\right. \\
& \left.-\Gamma^{(0)}\left(U_{\mathrm{l}, \mathrm{f}}(n)\right)-\Gamma^{(0)}\left(U_{\mathrm{r}, \mathrm{f}}(n)\right)\right] .
\end{aligned}
$$

In the following we will consider linear transport, $V \rightarrow$ 0 , in Coulomb blockade regime $E_{\mathrm{c}} \gg \varepsilon, k_{\mathrm{B}} T$, $\omega$, where only two channels (say $n$ and $n+1$ ) contribute appreciably to a given resonance conductance peak. The energies $U_{\nu, s}(n)$ and $U_{\nu, s}(n+1)$ given by (15) are proportional to the gate energy $\mu_{\mathrm{g}}=e\left(V_{\mathrm{g}}-V_{\mathrm{g}}^{\text {res }}\right)$ that measures the shift with respect to the gate resonance value $e V_{\mathrm{g}}^{\text {res }}=E_{\mathrm{c}}\left[2\left(n-n_{0}\right)+\right.$ 1]. The photoconductance can then be written in terms of a single rate $\Gamma^{(0)}\left(\mu_{\mathrm{g}}\right)$

$$
G\left(\mu_{\mathrm{g}}\right)=\frac{e^{2}}{2} \cdot \frac{\sum_{l= \pm} \Gamma^{(0)}\left(l \mu_{g}\right) \Gamma^{\prime}\left(-l \mu_{\mathrm{g}}\right)}{\sum_{l= \pm} \Gamma^{(0)}\left(l \mu_{\mathrm{g}}\right)},
$$

where $\Gamma^{\prime}(x) \equiv d \Gamma^{(0)}(x) / d x$. In the absence of the ac-field this reduces to the dc-case of the linear conductance 14

$$
G_{\mathrm{dc}}\left(\mu_{\mathrm{g}}\right)=\frac{e^{2}}{4} \cdot \frac{e^{-\beta \mu_{\mathrm{g}} / 2} \Gamma_{\mathrm{dc}}\left(\mu_{g}\right)}{k_{\mathrm{B}} T \cosh \left(\beta \mu_{\mathrm{g}} / 2\right)} .
$$

\section{Photoconductance}

In this section we analyze the behavior of the linear photoconductance in the discretization regime $k_{\mathrm{B}} T \ll \varepsilon$. In order to do this it is useful to recall the characteristic energy scales for the transport. The quantum dot is characterized by a ground state energy of $n$ charge $E_{0}(n)=E_{\mathrm{c}}\left(n-n_{\mathrm{g}}\right)^{2}$. The energy differences of the many body states of $n+1$ and $n$ electrons are

$$
\mu(n, l)=2 E_{\mathrm{c}}\left(n-n_{\mathrm{g}}+\frac{1}{2}\right)+l \varepsilon .
$$

Positive or negative integers $l$ denote the differences of the number of charge excitation quanta with the discretization (11). They do not change the number of particle in the dot. The quantity $\mu(n, l)$ plays the role of chemical potential of the dot and defines the transport region.

We start now to discuss the simpler case of large discretization energy $\varepsilon>\omega$. Here we expect that the collective charge excitation quanta cannot be excited by the photons, and only the ground state chemical potential 


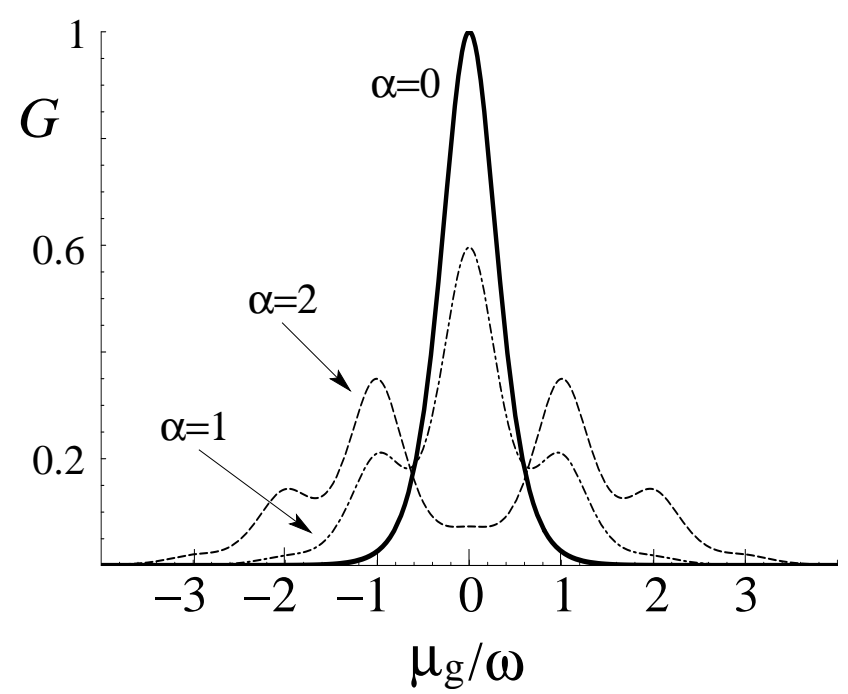

Fig. 1. Linear conductance, normalized to the maximum of the dc-resonance (thick line), in the non-interacting case $g=1$, as a function of the gate energy $\mu_{g} / \omega$. Different curves correspond to different values of $\alpha=e V_{\mathrm{ac}} / \omega$, for $\varepsilon / \omega=3.5, k_{\mathrm{B}} T=\omega / 5$, $\omega / \omega_{c}=10^{-4}$.

$\mu(n, l=0)$ will be involved. This is shown in Fig. 1. The conductance is plotted as a function of the gate energy $\mu_{\mathrm{g}}$ for different values of the parameter $\alpha=e V_{\mathrm{ac}} / \omega$ and $g=1$. Temperature is low enough $\left(k_{\mathrm{B}} T<\omega\right)$ in order to observe the discreteness of the photon energy. The curve for $\alpha=0$ corresponds to the dc-case $(30)$. Here the conduction is present only when $\mu(n, l=0)$ lines up with the Fermi levels of the leads. The width of the resonance is due to the finite temperature. Away from the voltage range characterizing the resonance peak, the system is affected by Coulomb blockade and does not conduct within the sequential tunneling regime. The transport properties radically change when we turn on an ac-field. By modulating the gate polarization $\alpha$ the conduction electrons exchange photons with the external field. The corresponding inelastic electron-photon scattering results in the splitting of the dc-resonance into a series of principal resonance sidebands. They show up at integer value of the frequency $\mu_{\mathrm{g}}=m \omega$ with $m= \pm 1, \pm 2 \ldots$

Increasing the frequency, transitions to excited charge mode states can also be supported. The corresponding structures in the conductance are shown in Fig. 2. This multi-level regime is characterized by resonance peaks due to the interplay of discrete charge quanta, with inelastic electron-photon scattering. Their energy positions are situated at $\mu_{\mathrm{g}}=m \omega+l \varepsilon$, with $m, l$ positive and negative integers. The middle curve of Fig. 2 2 shows the principal peaks for $m=0, \pm 1, \pm 2$, and the combinations between the lowest excited states $(l= \pm 1)$, and the ground state for $m= \pm 1, \pm 2$. Higher excited states contribute also because of the rational value $\varepsilon / \omega=2.5$. Decreasing this ratio (top figure) the number of peaks increase. The difference in their intensities is due to the modulation of the corresponding Bessel function driven by $\alpha$ (see later for a more detailed discussion).

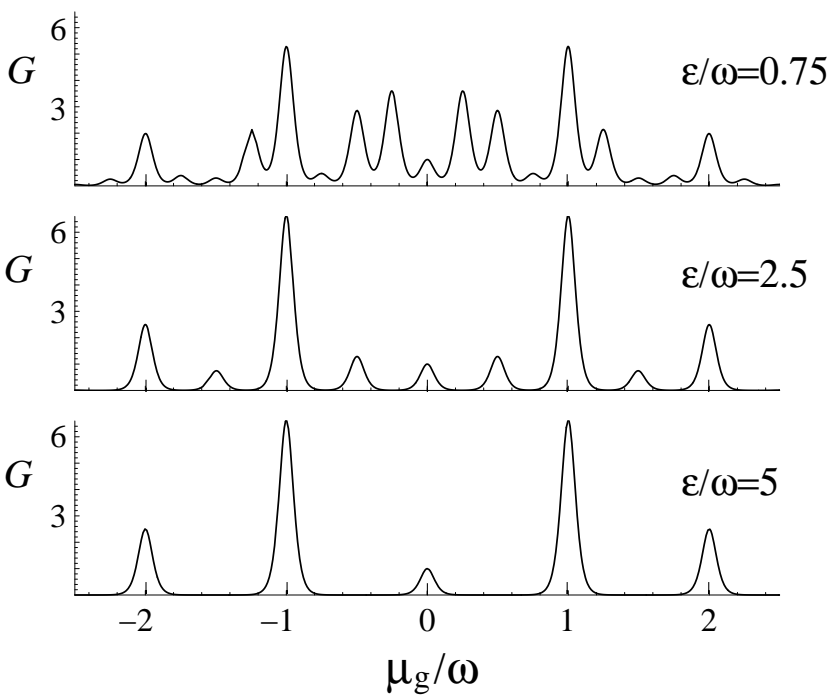

Fig. 2. Linear conductance, normalized to the value of the central resonance at $\mu_{g}=0$, as a function of the gate energy $\mu_{g} / \omega$, for $\alpha=2, g=1, k_{\mathrm{B}} T=\omega / 30, \omega / \omega_{c}=10^{-4}$ and different values of $\varepsilon / \omega$.

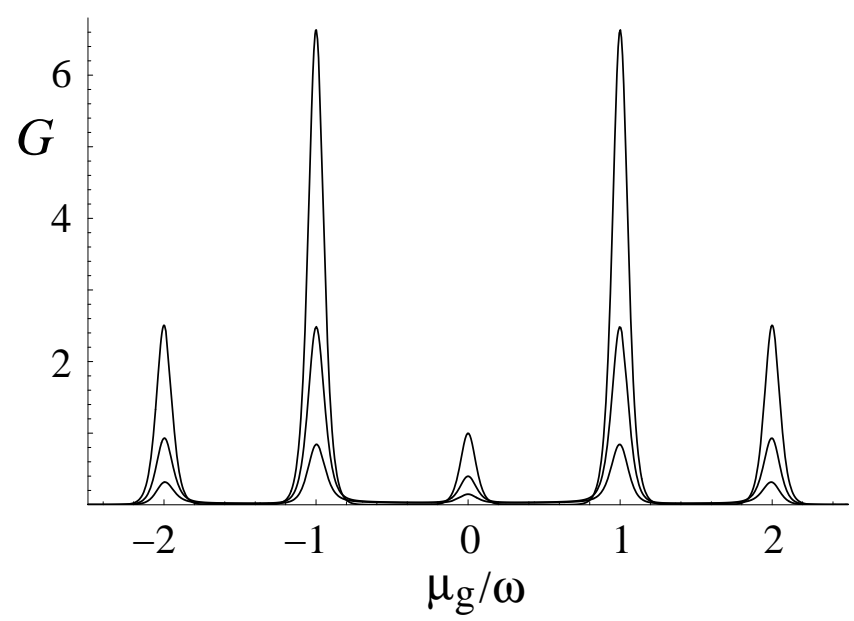

Fig. 3. Linear conductance, as a function of the gate energy $\mu_{g} / \omega$, for $\alpha=2, \varepsilon / \omega=5 / g, k_{\mathrm{B}} T=\omega / 30, \omega / \omega_{c}=10^{-4}$. The curves correspond to $g=1,0.95,0.9$ starting from the higher to the lower peak intensities. The curves are normalized to the value of the central resonance $\left(\mu_{\mathrm{g}}=0\right)$ for $g=1$.

In the presence of interaction $(g<1)$ the discretization energy is larger, $\varepsilon \propto 1 / g$ (cf. eq.(111)), then for a fixed frequency, increasing the interaction, we always end up into a regime where only the principal values are observable.

Figure 3 shows the conductance for relatively low interaction at fixed temperature, in the first regime $\varepsilon>\omega$. One can easily see that while the positions of the principal sidebands are not changed by the interactions, the intensities of the peak resonances are drastically reduced. Moreover, the stronger is the interaction the smaller is the resolution of photo spectroscopy one can perform. For $g<1$ the conductance assumes a smoother shape and the peaks become progressively less distinguishable. When lowering the temperature, the maxima of all of the resonance peaks 


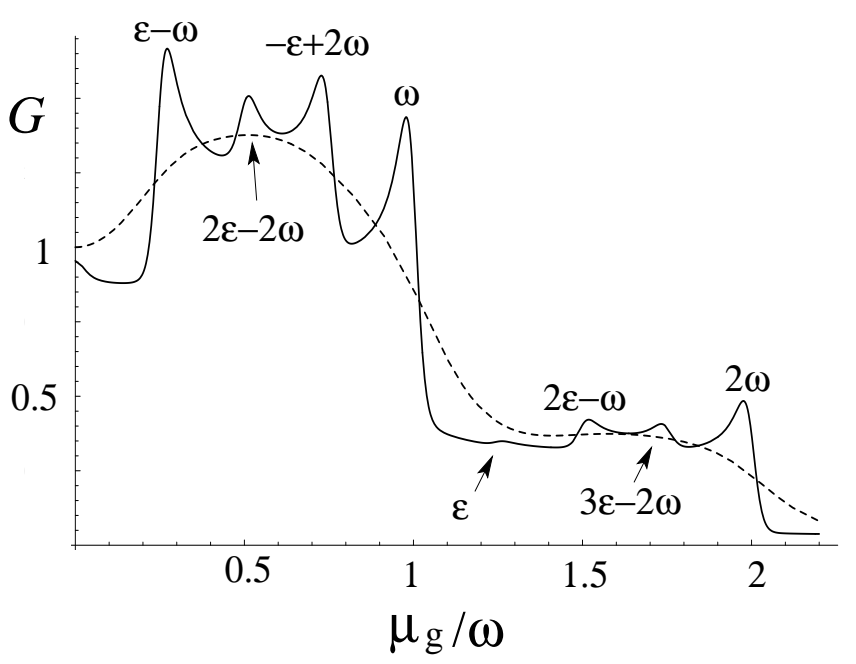

Fig. 4. Linear conductance as a function of $\mu_{\mathrm{g}} / \omega$, for $g=$ 0.6 and $k_{\mathrm{B}} T=\omega / 15$ (dashed line), $k_{\mathrm{B}} T=\omega / 100$ (full line) with $\alpha=2, \varepsilon / \omega=1.25$, and $\omega / \omega_{c}=10^{-4}$. The curves are normalized to the value of the central resonance $\left(\mu_{\mathrm{g}}=0\right)$ at $k_{\mathrm{B}} T=\omega / 15$.

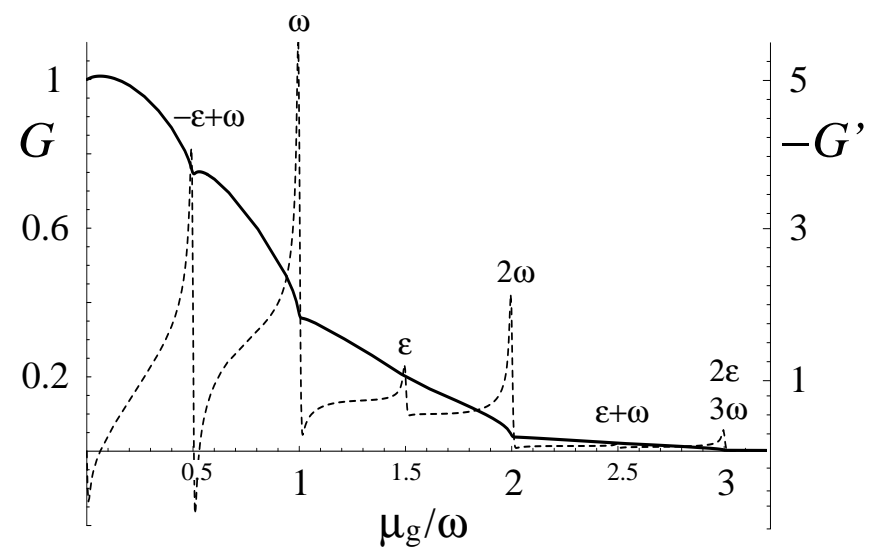

Fig. 5. Linear conductance (full line), and its derivative $G^{\prime}=$ $\omega \partial G / \partial \mu_{\mathrm{g}}$ (dashed line), in the strong interaction limit $g=0.4$, for $k_{\mathrm{B}} T=\omega / 500, \alpha=2, \varepsilon / \omega=1.5$, and $\omega / \omega_{c}=10^{-4}$. The curves are normalized to the value of the conductance at the central resonance $\left(\mu_{\mathrm{g}}=0\right)$.

scale according to a power law similar to the dc-case [14]

$$
G_{\max } \propto\left(k_{\mathrm{B}} T\right)^{1 / g-2} .
$$

This expression shows the interplay of temperature and interaction and strongly discriminates between two different regimes. In the weak interacting regime, characterized by $1 / 2<g \leq 1$, conductance persists in showing a peaklike behavior which can be enhanced decreasing temperature. On the other hands in the strong interacting regime $(g \leq 1 / 2)$ the ac-field is no longer able to split the dcresonance into a series of peaks. Figure 4 shows the conductance as a function of $\mu_{\mathrm{g}}$ for weak interaction $(g=0.6)$ and $\varepsilon \approx \omega$. The dashed and full curves correspond to higher and lower temperatures, respectively. Decreasing temperature, the power law increas of the peaks as in (32) allows to distinguish beween principal and excited states. From the latter one can deduce the interaction dependence of the plasmon modes.

Figure 5 shows the conductance and its derivative for strong interaction $g=0.4$. Here the peaks are replaced by a step-like behavior. Their positions depend as above by the combination $m \omega+l \varepsilon$. In correspondence of each step the derivative of the conductance presents sharp peaks. Their line-shape strongly depends on the interaction, with the maximum that scales in temperature as $\left(\partial G / \partial \mu_{\mathrm{g}}\right) \approx$ $\left(k_{\mathrm{B}} T\right)^{1 / g-3}$.

The absence of resonance peaks in the photoconductance is a behavior characteristic also of "metallic" quantum dots with a continuum of electronic states (discretization energy much smaller than the temperature) [22]. However, differently from our discrete case where this absence is present only for strong interactions $g \leq 1 / 2$, here is always present at any interaction $g \leq 1$. This is due to a sort of doubling of the interaction between discrete and continuum dots 13,14 .

Despite the positions and the shapes of the photoconductance peaks are modified by the interaction, the corresponding height normalized to the dc value, still follows a universal relation. For $k_{\mathrm{B}} T \ll \omega \ll \varepsilon$ and $g>1 / 2$, one can approximate the derivative of the rate (27) at $\mu_{\mathrm{g}}=m \omega$ as

$$
\Gamma^{\prime}(m \omega) \approx \Gamma^{\prime}(-m \omega) \approx J_{m}^{2}(\alpha) \Gamma_{\mathrm{dc}}^{\prime}(0),
$$

where $\Gamma_{\mathrm{dc}}^{\prime}(0)$ is the derivate of the dc-rate $(17)$ for $\mu_{\mathrm{g}}=0$. The photoconductance ratio assumes the universal form

$$
\frac{G\left(\mu_{\mathrm{g}}=m \omega\right)}{G_{\mathrm{dc}}\left(\mu_{\mathrm{g}}=0\right)} \approx J_{m}^{2}(\alpha) .
$$

Figure 6 represents the ratio (34), in the presence of interactions $(g=0.8)$, for $m=0$, and $m=1$ (inset) as a function of $\alpha$, at different temperatures. When the temperature is sufficiently low the conductance ratio approaches the Bessel function of the order of the corresponding principal sidebands. Such a relation allows to select and enhance a given sideband at the cost of the others. This can be achieved because the zeros of the Bessel functions appear at different values of $\alpha$. The modulation of the peaks can then be controlled independently of the interaction simply by changing the ac-external gate.

\section{Conclusions}

In summary, we have evaluated the linear photo-conductance of a $1 \mathrm{D}$ quantum dot imbedded in a non-Fermi liquid. We have considered the sequential tunneling regime at temperatures smaller than the discretization energy of the dot and the photon frequency. Varying the frequency we treated both the ground state transport and the transport via excited modes. The latter are due to the collective quantized plasmon modes in the dot, with an energy renormalized by the interaction. We have shown that the presence of the interactions dramatically changes the shape 


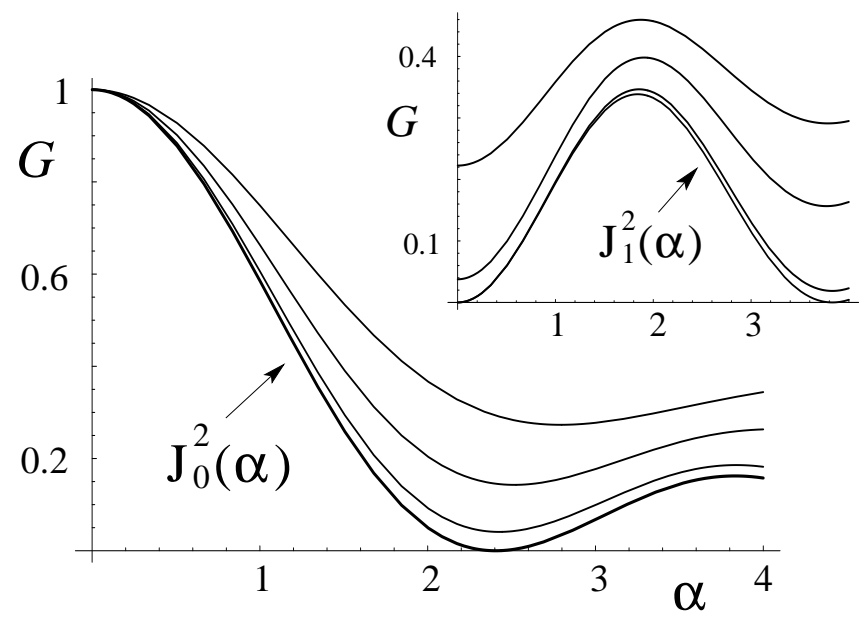

Fig. 6. Linear conductance, normalized to the height of the dc-resonance $(\alpha=0)$, as a function of $\alpha=e V_{\mathrm{ac}} / \omega$, for $\mu_{\mathrm{g}}=0$, and $\mu_{\mathrm{g}}=\omega$ (inset), with $\varepsilon / \omega=4.5, g=0.8$, and $\omega / \omega_{c}=10^{-4}$. Starting from the top to the bottom, the different curves correspond to temperatures $k_{\mathrm{B}} T / \omega=1 / 2,1 / 5,1 / 20$ (main figure), and to $k_{\mathrm{B}} T / \omega=1 / 3,1 / 5,1 / 50$ (inset). The curves indicated with arrows represent $J_{0}^{2}(\alpha)$ and $J_{1}^{2}(\alpha)$.

and the position of the exited peaks of the photoconductance with respect to the non-interacting case. We found two different transport regimes depending on the strength of the interaction. For weak interactions $1 / 2<g<1$ the linear conductance presents localized peaks. Their intensities scale at low temperatures with a non-Fermi liquid power law driven by the interactions. For strong interactions $g \leq 1 / 2$ the linear conductance keeps a compact step-like profile and hardly discriminates between the different sidebands. Peaks appear in this case in higher derivatives of the conductance with respect to the gate voltage.

In evaluating the photoconductance we have considered sequential tunneling processes in the limit of large barrier and high frequencies. We have calculated the photoconductance perturbatively in the tunneling matrix element $\Delta$. To be consistent with this last assumption the conductance has to be much smaller than $e^{2} / 2 \pi$, that of a clean wire . Considering for example the dc-conductance $G_{\mathrm{dc}}$ at the peak, this implies for the rate (c.f. eq (30))

$$
\Gamma_{\mathrm{dc}} \ll k_{\mathrm{B}} T .
$$

As pointed already out by Furusaki [13], this condition, when $g<1 / 2$, once it is satisfied at some high temperature, remains valid until $T \rightarrow 0$. On the other hands at $g>1 / 2$ there is always a critical temperature $k_{\mathrm{B}} T_{\mathrm{c}}=$ $\varepsilon(\Delta / \varepsilon)^{g /(2 g-1)}$ below which perturbation expansion fails. The ac-terms of the more general rate $\Gamma^{(0)}$ follow similar argument being connected to $\Gamma_{\mathrm{dc}}$ via Bessel functions. Note that because we always considered temperatures smaller than the frequencies the relation (35) automatically satisfies the requirement of high frequencies $\omega \gg \Gamma^{(0)}$.

The constraint (35) has to be considered together with the condition that coherent processes, like cotunneling, are negligible with respect to the sequential ones. Indeed at low enough temperatures one would expect that coherent tunneling processes could dominate, especially in the region away from resonance where sequential tunneling gives an almost negligible contribution. In [13] one can find an exhaustive discussion of the interplay between cotunneling and sequential tunneling for dc-transport. In particular it is shown that as long as (35) is satisfied the sequential processes are the dominant ones near to the peak resonance. Cotunneling contribution starts to become important in the tail of the peak.

Another important point concerns the spin dynamics. In the present paper we considered a spinless electron gas, concentrating only on the charge contribution. This was partly motivated by the spin-charge separation effect present in a Luttinger liquid. However we expect that the presence of the spin will introduce novel ac-effects. In particular the previous $g$ factor will be renormalized to an effective $g_{\text {eff }}$ given by $2 / g_{\text {eff }}=1 / g+1 / g_{\sigma}$. The last term represents the contribution of the spin. For zero exchange interactions $g_{\sigma}=1$. Moreover, we will have spin and charge addition and discretization energies [15]. This will enlarge the possible ac-transport channels involving more peaks in the photoconductance due to the spin. This interesting topics will be the subject of future work.

We expect that the several above effects due to the interplay between interaction and ac-fields can be observed in the future in the transport through double barrier in cleaved-edge-overgrowth quantum wires [4], and in carbon nanotubes [36.

This work has been supported by EU within TMR and RTN programmes, by Italian MURST via PRIN 2000.

\section{References}

1. S. Tarucha, T. Honda, and T. Saku, Solid State Commun. 94, 413 (1995).

2. A. Yacoby, H.L. Stormer, N.S. Wingreen, L.N. Pfeiffer, K.W. Baldwin, and K.W. West, Phys. Rev. Lett. 77, 4612 (1996).

3. A. Yacoby, H.L. Stormer, K.W. Baldwin, L.N. Pfeiffer, and K.W. West, Solid State Commun. 101, 77 (1997).

4. O.M. Auslaender, A. Yacoby, R. de Picciotto, K.W. Baldwin, L.N. Pfeiffer, and K.W. West, Phys. Rev. Lett. 84, 1764 (2000).

5. M. Sassetti, and B. Kramer, Phys. Rev. Lett. 80, 1485 (1998); Eur. Phys. J. B 4, 357 (1998).

6. J. M. Luttinger J. Math. Phys. 4, 1154 (1963).

7. F.D.M. Haldane, J. Phys. C 14, 2585 (1981).

8. J. Voit, Rep. Prog. Phys. 57, 977 (1995).

9. C.L. Kane, and M.P.A. Fisher, Phys. Rev. B 46, 15233 (1992).

10. A. Furusaki, and H. Nagaosa, Phys. Rev. B 47, 3827 (1993).

11. M. Sassetti, F. Napoli, and U. Weiss, Phys. Rev. B 52, 11213 (1995).

12. H. Maurey, and T. Giamarchi, Europhys. Lett. 38, 681 (1997).

13. A. Furusaki, Phys. Rev. B 57, 7141 (1998) 7141. 
14. A. Braggio, M. Grifoni, M. Sassetti, and F. Napoli, Europhys. Lett. 50, 236 (2000).

15. T. Kleimann, M. Sassetti, B. Kramer, and A. Yacoby, Phys. Rev. B 62, 8144 (2000).

16. A. Braggio, M. Sassetti, and B. Kramer, Phys. Rev. Lett. 87, 146802 (2001).

17. P.K. Tien, and J.P. Gordon, Phys. Rev. 129, 647 (1963).

18. D. Sokolovski, Phys. Rev. B 37, 4201 (1988).

19. C. Bruder, and H. Schöller, Phys. Rev. Lett. 72, 1076 (1994).

20. Ph. Brune, C. Bruder, and H. Schoeller, Physica E 1, 216 (1997).

21. L.P. Kouwenhoven, S. Jauhar, J. Orenstein, P.L. McEuen, Y. Nagamune, J. Motohisa, and H. Sakaki, Phys. Rev. Lett. 73, 3443 (1994).

22. L.P. Kouwenhoven, S. Jauhar, K. McCormick, D. Dixon, P.L. McEuen, Yu. V. Nazarov, N.C. van der Vaart, and C.T. Foxon, Phys. Rev. B 50, 2019 (1994).

23. R.H. Blick, R.J. Haug, D.W. van der Weide, K. von Klitzing, and K. Eberl, Appl. Phys. Lett. 67, 3924 (1995).

24. T.H. Oosterkamp, L.P. Kouwenhoven, A.E.A. Koolen, N.C. van der Vaart, and C.J.P.M. Harmans, Phys. Rev. Lett. 78, 1536 (1997).

25. T.H. Oosterkamp, T. Fujisawa, W.G. van der Wiel, K. Ishibashi, R.V. Hijman, S. Tarucha, and L.P. Kouwenhoven, Nature 395, 873 (1998).

26. M.H. Pedersen, M. Büttiker, Phys. Rev. B 58, 12993 (1998).

27. H. Qin, F. Simmel, R.H. Blick, J.P. Kotthaus, W. Wegscheider, and M. Bichler, Phys. Rev. B 63, 035320 (2001).

28. T. Fujisawa, and S. Tarucha, Jpn. J. Appl. Phys. 36, 4000 (1997).

29. M. Sassetti, U. Weiss, and B. Kramer, Solid State Commun. 97, 605 (1996).

30. A. Fechner, M. Sassetti, and B. Kramer, Europhys. Lett. 45, 693 (1999); A. Fechner, M. Sassetti, B. Kramer, and E. Galleani, Phys. Rev. B. in press (2001).

31. G. Cuniberti, A. Fechner, M. Sassetti, and B. Kramer, Europhys. Lett. 48, 66 (1999).

32. C.W.J. Beenakker, Phys Rev. B 44, 1646 (1991).

33. G.L. Ingold, and Y.V. Nazarov, in: Single Charge Tunneling, NATO ASI B 294 p.21, edited by H. Grabert, and M.H. Devoret, (Plenum, New York, 1992).

34. M. Sassetti, and B. Kramer, Phys. Rev. B 54, R5203 (1996).

35. M. Grifoni, M. Sassetti, and U. Weiss, Phys. Rev. E 53, R2033 (1996).

36. H.W.Ch. Postma, M. de Jonge, Z. Yao, and C. Dekker Phys. Rev. B 62, R10653 (2000). 